\title{
Ritter-Type lodo(III)amidation of Unactivated Alkynes for the Stereoselective Synthesis of Multisubstituted Enamides
}

\author{
Jinkui Chai, ${ }^{[a, b,+]}$ Wei Ding, ${ }^{[b, c,+]}$ Shingo Ito, ${ }^{[b]}$ Junliang Wu, ${ }^{*[a]}$ and Naohiko Yoshikai* ${ }^{[b, d]}$
}

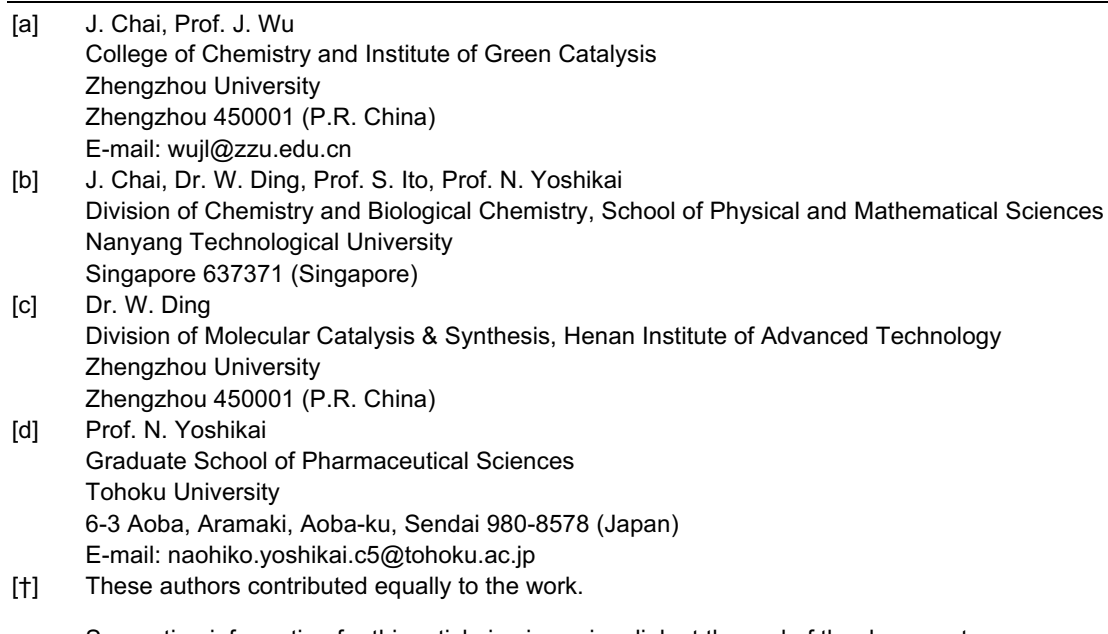

Supporting information for this article is given via a link at the end of the document.

\begin{abstract}
The Ritter reaction, Brønsted- or Lewis acid-mediated amidation of alkene or alcohol with nitrile via a carbocation, represents a classical method for the synthesis of tertiary amides. Although analogous reaction through a vinyl cation or a species alike may offer a route to enamide, an important synthetic building block as well as a common functionality in bioactive compounds, such transformations remain largely elusive. Herein, we report on a Ritter-type transdifunctionalization of alkynes with trivalent iodine electrophile and nitrile, which affords $\beta$-iodanyl enamides in moderate to good yields. Mediated by benziodoxole triflate (BXT), the reaction proves applicable to a variety of internal alkynes as well as to various alkyland arylnitriles. The benziodoxole group in the product serves as a versatile handle for further transformations, thus allowing for the preparation of various tri- and tetrasubstituted enamides that are not readily accessible by other means.
\end{abstract}

Enamides are versatile intermediates for the synthesis of nitrogen-containing molecules. ${ }^{[1]}$ As stable and electron-rich olefins, they participate in diverse transformations such as asymmetric hydrogenation, ${ }^{[2]}$ cycloaddition, ${ }^{[1 a, b]}$ addition to carbonyls and imines, ${ }^{[3]}$ and halofunctionalization. ${ }^{[4]}$ As for transition metal-catalyzed $\mathrm{C}-\mathrm{C}$ coupling, enamides have been extensively used in Heck reaction, and more recently have attracted attention as substrates for $\mathrm{C}-\mathrm{H}$ activation ${ }^{[5]}$ and enantioselective hydroalkynylation. ${ }^{[6]}$ Enamide moiety is also prevalent in bioactive natural products as well as in drug candidates. ${ }^{[1]}$ Conventional methods for the enamide synthesis include condensation of amides and aldehydes, Curtius rearrangement of unsaturated acyl azides, and Wittig or HornerWadsworth-Emmons olefination, which often suffer from narrow scope, low stereocontrol, and/or harsh reaction conditions. ${ }^{[7]}$ More recently, methods based on the functionalization of $\mathrm{C} \equiv \mathrm{C}$ bonds have been developed, such as hydroamidation to alkynes ${ }^{[8]}$ and carbometalation to ynamides, ${ }^{[9]}$ enabling stereocontrol over the enamide $\mathrm{C}=\mathrm{C}$ bond. ${ }^{[10]}$ However, the former method is currently limited to terminal alkynes and inherently does not allow access to tetrasubstituted enamides, whereas the latter requires specific types of amide moieties to promote the desired carbometalation. The Cu-catalyzed amidation of alkenyl halides represents another well-established stereoselective route to enamides, ${ }^{[11]}$ but the synthesis of the prerequisite, stereodefined alkenyl halides hinders its application to highly substituted enamides. As such, the chemical space of tetrasubstituted enamides, especially those containing biologically more relevant secondary amide moieties, remains largely inaccessible. ${ }^{[12]}$

The Ritter reaction, Brønsted- or Lewis acid-mediated reaction of olefins or alcohols with nitrile via carbocation intermediates, represents a classical but important synthetic approach to tertiary alkyl amides (Scheme 1a). ${ }^{[13]}$ In analogy to the Ritter amidation, one would conceive a possible approach to enamides involving the addition of nitrile to acid- or electrophileactivated alkyne as a vinyl cation-like species, followed by capture of the nitrilium intermediate with water (Scheme 1b). Despite the apparent simplicity, to our knowledge, such transformation on simple unactivated alkynes has remained elusive, while Liu and Yang recently reported a gold-catalyzed Ritter-type amidation of chloro- and bromoalkynes to give trisubstituted, $\beta$ haloenamides. ${ }^{[14]}$ Herein, we report that benziodoxole triflate (BXT; 1) ${ }^{[15]}$ a cyclic trivalent iodine electrophile, promotes the Ritter-type difunctionalization of unactivated alkynes to afford tetrasubstituted $\beta$-iodanyl enamides in a regio- and stereoselective fashion (Scheme 1c). The reaction tolerates a variety of internal alkynes as well as alkyl- and arylnitriles. The iodanyl group in the product serves as a handle for the synthesis 
of tri- and tetrasubstituted enamides that are otherwise difficult to access by existing methods.

(a) Ritter reaction: Well studied

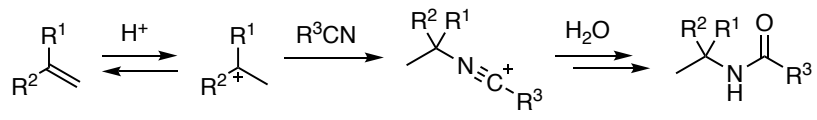

(b) Ritter-type functionalization of alkyne: Elusive

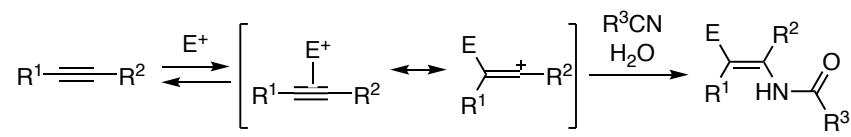

(c) This work: Ritter-type iodo(III)amidation of unactivated alkynes

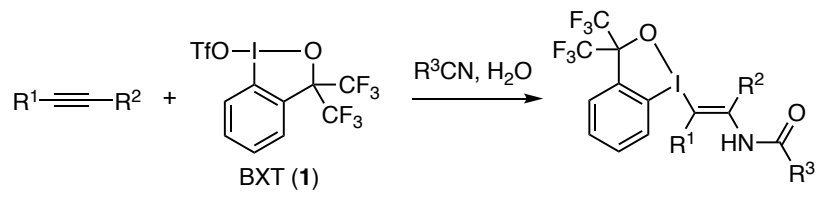

- trans-selective difunctionalization

- various internal alkynes and nitriles

- $\beta$-iodanylenamide to tri- and tetrasubstiteud enamides

Scheme 1. Ritter-type amidation.

Recently, we reported a trans-iodo(III)etherification reaction of alkynes with BXT and alcohol or with $\mathrm{BF}_{3}$-activated fluorobenziodoxole (FBX) and dialkyl ether, which allowed for the stereoselective synthesis of multisubstituted vinyl ethers. ${ }^{[16]}$ During this study, we failed in engaging primary and secondary amides as nucleophiles in place of alcohol, observing no desired enamide product. Given this result, we turned our attention to the use of nitrile and water to achieve the putative iodo(III)amidation. In fact, trivalent iodine-mediated functionalization of alkynes with nitrile and water has a precedent. Saito and coworkers reported a synthesis of oxazoles from alkyne, nitrile, and water mediated by iodosylbenzene (PhIO) and a Brønsted acid such as $\mathrm{TfOH}$ (Scheme 2a). ${ }^{[17]}$ However, their mechanistic study indicated that this reaction was initiated by the addition of triflate anion, rather than nitrile, to the I(III)-activated alkyne, and in fact no iodanylsubstited enamide intermediate was observed. During our study on the iodo(III)etherification, we also observed oxazole formation from 1-phenyl-1-butyne and $\mathrm{FBX} / \mathrm{BF}_{3}$ in wet, HPLC-grade acetonitrile, which was assumed to involve the addition of the nitrile to the BX-activated alkyne (Scheme 2b). Despite these precedent and observation, given the stability of the BX group among other iodanyl groups, ${ }^{[18]}$ we surmised that the desired Ritter-type iodo(III)amidation would be feasible under careful control of water content and other conditions.

Upon extensive screening of reaction conditions (see Tables S1 and S2), we succeeded in the desired iodo(III)amidation of 4octyne (2a) with $\mathbf{1}$, acetonitrile, and water under two sets of optimized conditions (Scheme 2c). In conditions $\mathrm{A}, \mathbf{2 a}$ was reacted with 1 (2 equiv) and $\mathrm{H}_{2} \mathrm{O}$ (1 equiv) in the presence of $\mathrm{Na}_{2} \mathrm{CO}_{3}$ (3 equiv) in dry MeCN (3a) as the solvent at room temperature, affording the desired trans-enamide 4 aa in $90 \%$ yield with exclusive stereoselectivity. Alternatively, conditions B employed 20 equiv of $\mathrm{MeCN}$ in 1,1,1,3,3,3-hexafluoroisopropanol (HFIP), which also efficiently promoted the reaction to afford 4aa in $88 \%$ yield. The yield dropped significantly in trifluoroethanol (TFE), and no desired product was obtained in other solvents such as DCE and toluene. Thus, HFIP appeared to benefit the reaction through its cation-stabilizing nature and low nucleophilicity. ${ }^{[19]}$ The molecular structure of 4 aa was unambiguously established by $\mathrm{X}$-ray crystallographic analysis.

(a) Saito et al. (ref 15)

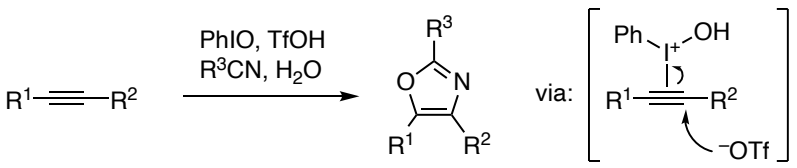

(b) Our preliminary observation

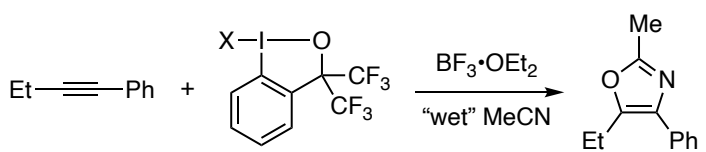

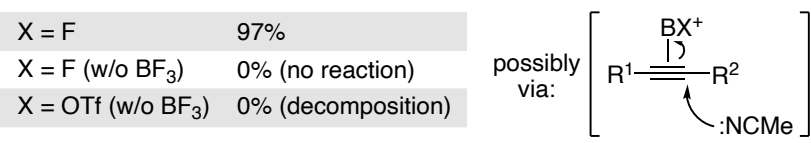

(c) lodo(III)acetamidation by BXT

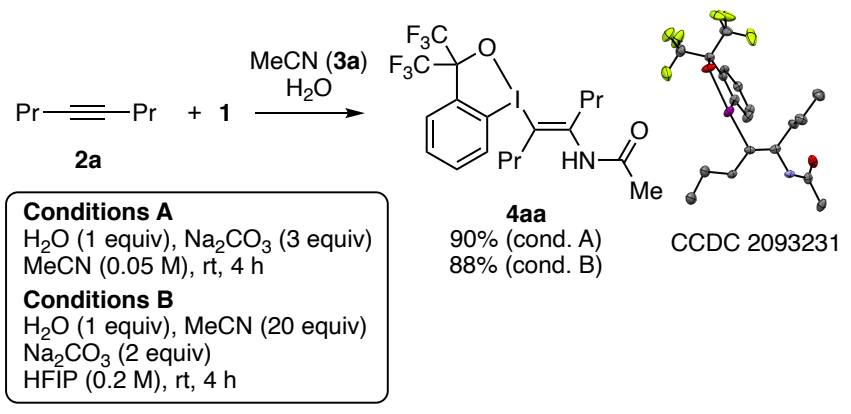

Scheme 2. lodine(III)-mediated reaction of alkyne with nitrile and water.

Table 1 summarizes the scope of alkynes for the iodo(III)acetamidation using $\mathrm{MeCN}$ and $\mathrm{H}_{2} \mathrm{O}$. A variety of dialkylalkynes smoothly participated in the reaction under either conditions A or B to afford the corresponding $\beta$-iodanylenamides 4aa-4ia in moderate to good yields. The preparation of 4aa could be performed on a $2 \mathrm{mmol}$ scale, albeit in somewhat lower yield. For the reactions of unsymmetrically substituted dialkylalkynes, amidation took place preferentially at the sterically more hindered acetylenic carbon, while the degree of steric discrimination was moderate, with regioisomer ratio of ca. $2: 1$ to $3: 1$, across alkynes containing methyl group at one end and primary or secondary alkyl group at the other end (see 4ea-4ha). Exclusive regioselectivity was observed in the reaction of 2,2-dimethyloct-3yne, where the amidation occurred at the carbon proximal to the tert-butyl group (see 4ia and its X-ray structure). Interestingly, this regioselectivity trend marks a sharp contrast with that observed in the iodo(III)etherification of dialkylalkynes with $\mathrm{MeOH}$, where the addition of methoxy group occurred selectively at the sterically less hindered position. ${ }^{[16 a]}$ This difference between the iodo(III)amidation and the iodo(III)etherification would be ascribed to the sterically less sensitive nature of the nitrile nucleophile compared to the alcohol nucleophile. As such, the reaction would have taken place to avoid the steric repulsion between the BX cation and the alkyne substituent rather than between the nitrile and the alkyne substituent. Besides dialkylalkynes, a series of aryl(alkyl)alkynes underwent iodo(III)acetamidation. Thus, 
amidation expectedly occurred at the position proximal to the cation-stabilizing aryl group, thus affording the corresponding enamide products $\mathbf{4 j a - 4 p a ~ i n ~ m o d e r a t e ~ y i e l d s . ~ A s ~ a ~ g e n e r a l ~ t r e n d , ~}$ conditions A proved suitable for alkynes bearing electrondonating or neutral substituent; they reacted much more sluggishly under conditions B. The opposite was the case for alkynes bearing electron-withdrawing substituents such as ester, ketone, and $\mathrm{CF}_{3}$ groups. An enyne could also be transformed into dienamide $4 \mathrm{qa}$ in $65 \%$ yield under conditions $\mathrm{A}$. Note that terminal alkynes failed to give the iodo(III)amidation product under the present conditions.

Table 1. lodo(III)acetamidation of various alkynes ${ }^{[a]}$

$$
\mathrm{R}^{1}=\mathrm{R}^{2}+\mathrm{CF}_{3} \stackrel{\mathrm{MeCN}, \mathrm{H}_{2} \mathrm{O}}{\mathrm{CF}_{3}} \stackrel{\text { Conditions A or B }}{\longrightarrow}
$$

$$
\sum_{\mathrm{NHAc}}^{\mathrm{Pr}}
$$

4aa, $85 \%(72 \%)^{[b]}(A) ; \quad$ 4ba, $81 \%(A) ; 88 \%(B) \quad$ 4ca, $80 \%(A) ; 83 \%(B)$ $87 \%(B)$<smiles>CC(Cl)C(NC(=O)Cl)=C(Cl)Cl</smiles><smiles>CCC(NC(C)=O)=C(C)Br</smiles>

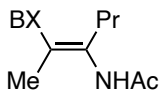

4da, 42\% (A); 75\% (B) 4ea, 74\%, rr =2.3:1 (A); 4fa, 71\%, rr $=2.0: 1(\mathrm{~A})$ $83 \%, r r=1.9: 1(B) \quad 78 \%, r r=2.0: 1(B)$<smiles>CC(Br)=C(C)NC(C)C</smiles><smiles>CC(=O)NC(C)=C(C)Br</smiles>

4ga, 73\%, rr $=3.4: 1(\mathrm{~A}) ; \quad$ 4ha, $74 \%, r r=2.2: 1(\mathrm{~A})$ $82 \%, r r=2.5: 1(B)$ $82 \%, r r=1.7: 1(B)$
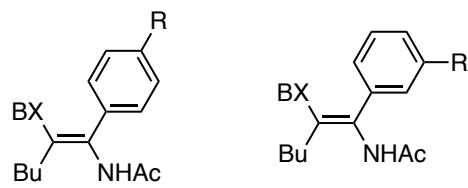

4ja $(R=O M e), 42 \%(A) \quad$ 4oa $(R=O M e), 39 \%(A)$ 4ka $(R=M e), 33 \%(A) \quad$ 4pa $\left(R=\mathrm{CO}_{2} \mathrm{Et}\right), 42 \%(B)$ 4la $\left(\mathrm{R}=\mathrm{CO}_{2} \mathrm{Et}\right), 51 \%(\mathrm{~B})$ $4 \mathrm{ma}(\mathrm{R}=\mathrm{COMe}), 51 \%(\mathrm{~B})$ 4na $\left(R=\mathrm{CF}_{3}\right), 39 \%(\mathrm{~B})$

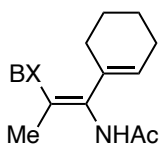

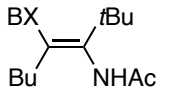
4ia, $44 \%(B)$

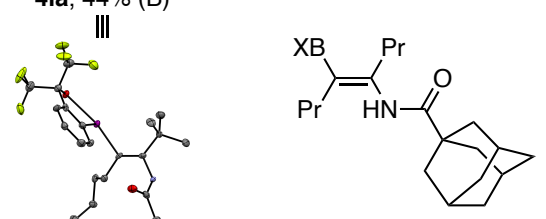

4am, $26 \%$ to the reaction but used as received. Attempted reactions using unpurified propionitrile or butyronitrile as the solvent under conditions $A$ afforded only low yields of the desired enamide, presumably due to the water content in such reagent-grade nitriles. Aromatic nitriles including parent and substituted benzonitriles and thiophene-2-carbonitrile also participated in the reaction with $\mathbf{2 a}$ to afford the desired products $4 \mathbf{a n}-\mathbf{4 a r}$ in moderate yields.

Table 2. lodo(III)amidation of 4-octyne with various nitriles ${ }^{[a]}$

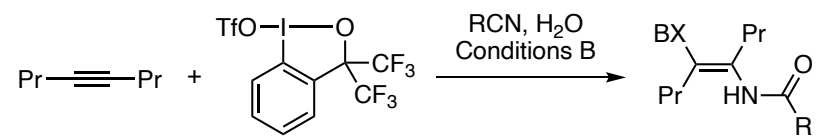

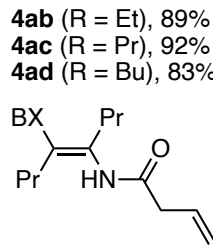

$4 q a, 65 \%(A)$

[a] The reaction was performed on a $0.2 \mathrm{mmol}$ scale. The symbol BX in the product formula refers to the benziodoxole moiety. For reactions that produced a mixture of regioisomers, the major isomer is shown along with $\mathrm{rr}$ (regioisomer ratio) determined by ${ }^{1} \mathrm{H}$ NMR. [b] The yield of a 2 -mmol scale reaction (reaction time $=8 \mathrm{~h}$ ) is given in the parentheses.

We next explored the scope of nitriles in the iodo(III)amidation of 4-octyne (Table 2). Here, conditions B proved effective across a wide variety of organonitriles. Thus, aliphatic nitriles including deuterated acetonitrile ([D $]$-4aa), primary alkyl nitriles (4ab-4ae), benzyl and allyl nitriles (4af-4ah), secondary alkyl nitriles (4ai4ak), and tertiary alkyl nitriles (4al and 4am) underwent the desired iodo(III)amidation to afford the corresponding $\beta$-iodanyl enamides in moderate to high yields. The yields were relatively insensitive to the steric size of the nitrile substituent, except that 1-adamanyl nitrile afforded the product $\mathbf{4 a m}$ in a modest $26 \%$ yield. In these reactions, unlike the iodo(III)acetamidation with $\mathrm{MeCN}$, the nitrile substrates were neither dried nor purified prior<smiles>[R6]C(=O)NC([R6])=C([R])Br</smiles><smiles>[R]C(=O)NC([18O])=C([R])Br</smiles>

$\left[D_{3}\right]-4 a a, 67 \%$

4ag, $58 \%$<smiles>[R7]C([R17])=C([PH])NC(=O)C(C)C</smiles><smiles>[R17]C(Br)=C(CCC)NC(=O)C1CC1</smiles>

4aj $(n=1), 71 \%$ 4ak $(n=3), 65 \%$

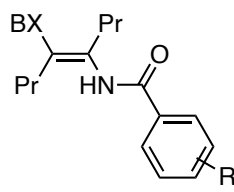

4an $(R=H), 46 \%$

4ao $(R=4-O M e), 45 \%$

4ap $(\mathrm{R}=4-\mathrm{Cl}), 54 \%$<smiles>[R]C(Br)=C(CCC)NC(=O)CC1CC1</smiles>

4ae, $71 \%$<smiles>[R]C(Br)=C(CCC)NC(=O)CC1=CCCCC1</smiles>

4ah, $66 \%$<smiles>[R7]C(Br)=C(CCC)NC(=O)C(C)(C)C</smiles>

4al, $67 \%$

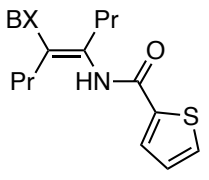

$4 a r, 54 \%$ product formula refers to the benziodoxole moiety.

The product of the present reaction, $\beta$-iodanyl enamide, provides opportunities for further synthetic transformations via cleavage of the BX group (Scheme 3a). ${ }^{[16 a, 18 d, 20,21]}$ Sonogashira and Stille couplings of $\mathbf{4 a a}$ afforded the conjugate enamides $\mathbf{5}$ and 6, respectively, in good yields. While Sonogashira coupling took place with retention of the olefin stereochemistry, Stille coupling afforded a mixture of $E / Z$ isomers in ca. 5:1 ratio. CuCN/prolinemediated cyanation of 4 aa proceeded smoothly at $105{ }^{\circ} \mathrm{C}$ to afford the products $(E)-7$ and $(Z)-7$ as separable isomers in ca. 3:1 ratio. At a lower temperature of $50{ }^{\circ} \mathrm{C}$, the reaction furnished the $\beta$-iodoenamide 8 with $E / Z$ ratio of $5: 1$. Hydrodehalogenation of 4 aa with $\mathrm{Pd} /$ formic acid system afforded the trisubstituted enamide $9(E / Z=5: 1)$ in good yield, whereas reductive homocoupling of 4aa mediated by $\mathrm{Pd}$ catalyst and $\mathrm{Zn}$ afforded the novel diene-diamide $10(E / Z=4: 1)$ in excellent yield. It should be pointed out that, unlike the partial loss of the stereochemical integrity in the $\mathrm{Pd}$-catalyzed coupling reactions of $4 \mathbf{a a}$, analogous [a] The reaction was performed on a $0.2 \mathrm{mmol}$ scale. The symbol BX in the 
reactions of $\beta$-iodanyl vinyl ether took place with retention of the stereochemistry. ${ }^{[16 a]}$ While the reason for this difference remains unclear, we speculate that the vinylpalladium species formed upon oxidative addition of $\mathbf{4 a a}$ has a partial carbene-like character due to the electron-donating effect of the amide group, thus allowing rotation of the olefinic $\mathrm{C}-\mathrm{C}$ bond. The chelating ability of the amide group might also facilitate the isomerization of the vinylpalladium species.

Many of the multisubstituted enamides synthesized above are not readily accessible by other means, and hence would open numerous opportunities for further synthetic transformations. For demonstration, we explored the application to the pyridine/pyrimidine synthesis developed by Movassaghi (Scheme 3b). ${ }^{[22]}$ Thus, trisubstituted enamides obtained through the iodo(III)amidation/hydrodehalogenation sequence were subjected to $\mathrm{Tf}_{2} \mathrm{O}$, 2-chloropyridine, and electron-rich alkyne or nitrile, affording multisubstituted pyridines 11-14 and pyrimidine 15 in good yields.

(a) Transformation of C-I(III) bond

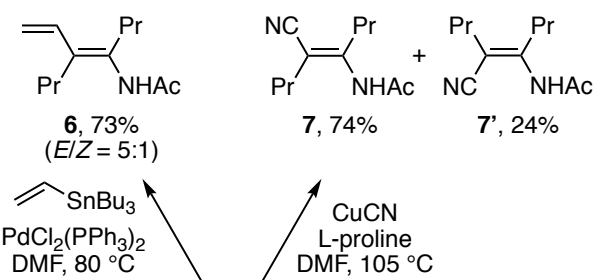

$$
\text { (E) }
$$

(b) Application to Movassaghi pyridine/pyrimidine synthesis<smiles>[R3]C(=O)NC([R])=C([R7])[R]</smiles>

$11,81 \%$

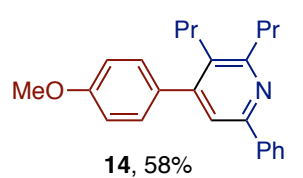

$14,58 \%$<smiles>[R7][Y]1=C([R])N=C([R])[Y]([H])C1[R]</smiles>

$$
\mathrm{X}=\mathrm{CH} \text { or } \mathrm{N}
$$

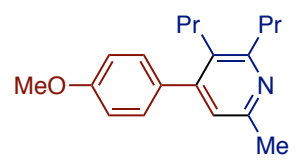

12, $73 \%$

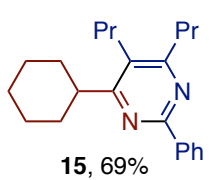

$$
\underset{\mathrm{R}^{4}-\mathrm{C} \equiv \mathrm{N}}{\mathrm{R}^{4} \underset{\mathrm{or}}{=} \mathrm{H}} \stackrel{\mathrm{Tf}_{2} \mathrm{O}, 2-\mathrm{CIPy}}{\mathrm{CH}_{2} \mathrm{Cl}_{2},-78^{\circ} \mathrm{C} \text { to rt }}
$$

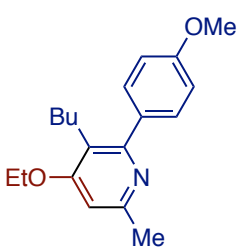

$13,65 \%$
Scheme 3. Follow-up transformations of $\beta$-iodanyl enamides
In conclusion, we have developed a Ritter-type transdifunctionalization of alkynes mediated by trivalent iodine electrophile BXT for the synthesis of multisubstituted enamides. The reaction was achieved with careful choice of the reaction conditions including the water content and the reaction medium, and proved applicable to a variety of internal alkynes as well as nitriles, thus affording trans-iodanyl enamides in moderate to good yields. The versatility of the BX group allows for the preparation of various tri- and tetrasubstituted enamides through cross-coupling. The multisubstituted enamides made accessible by the present method would inspire further development of enamide-based transformations for the synthesis of nitrogencontaining molecules.

\section{Acknowledgements}

This work was supported by the Agency for Science, Technology and Research (A*STAR) AME IRG Grant (A2083c0056). J.C. thanks China Scholarship Council for the support of overseas visiting student program. We thank Dr. Yongxin Li (Nanyang Technological University) for his assistance with the X-ray crystallographic analysis.

Keywords: enamides $•$ alkynes $•$ amidation $\bullet$ hypervalent iodine compounds $\cdot$ nitriles

[1] a) T. Courant, G. Dagousset, G. Masson, Synthesis 2015, 47, 17991826; b) G. Bernadat, G. Masson, Synlett 2014, 25, 2842-2867; c) D. R. Carbery, Org. Biomol. Chem. 2008, 6, 3455-3460.

[2] K. Gopalaiah, H. B. Kagan, Chem. Rev. 2011, 111, 4599-4657

[3] R. Matsubara, S. Kobayashi, Acc. Chem. Res. 2008, 41, 292-301.

[4] A. Alix, C. Lalli, P. Retailleau, G. Masson, J. Am. Chem. Soc. 2012, 134, 10389-10392.

[5] T. Zhu, S. Xie, P. Rojsitthisak, J. Wu, Org. Biomol. Chem. 2020, 18, 1504-1521.

[6] a) X.-Y. Bai, Z.-X. Wang, B.-J. Li, Angew. Chem. Int. Ed. 2016, 55, 90079011; b) X.-Y. Bai, W.-W. Zhang, Q. Li, B.-J. Li, J. Am. Chem. Soc. 2018, 140, 506-514; c) S.-L. Zhang, W.-W. Zhang, B.-J. Li, J. Am. Chem. Soc. 2021, 143, 9639-9647.

[7] G. Evano, A. C. Gaumont, C. Alayrac, I. E. Wrona, J. R. Giguere, O. Delacroix, A. Bayle, K. Jouvin, C. Theunissen, J. Gatignol, A. C. Silvanus, Tetrahedron 2014, 70, 1529-1616.

[8] L. B. Huang, M. Arndt, K. Goossen, H. Heydt, L. J. Goossen, Chem. Rev. 2015, 115, 2596-2697.

[9] a) B. Gourdet, H. W. Lam, J. Am. Chem. Soc. 2009, 131, 3802-3803; b) Y. Minko, M. Pasco, H. Chechik, I. Marek, Beilstein J. Org. Chem. 2013, 9, 526-532.

[10] For enamide synthesis through other types of functionalization of $C \equiv C$ bonds, see: a) Mulder, J. A.; Kurtz, K. C. M.; Hsung, R. P.; Coverdale, H.; Frederick, M. O.; Shen, L.; Zificsak, C. A. Org. Lett. 2003, 5, 15471550; b) Li, M.; Yuan, H.; Zhao, B.; Liang, F.; Zhang, J. Chem. Commun. 2014, 50, 2360-2363; c) Ide, M.; Yauchi, Y.; Iwasawa, T. Eur. J. Org. Chem. 2014, 3262-3267.

[11] T. Kuranaga, Y. Sesoko, M. Inoue, Nat. Prod. Rep. 2014, 31, 514-532.

[12] B. M. Trost, J. J. Cregg, N. Quach, J. Am. Chem. Soc. 2017, 139, 51335139.

[13] a) A. Guerinot, S. Reymond, J. Cossy, Eur. J. Org. Chem. 2012, 19-28; b) D. H. Jiang, T. He, L. Ma, Z. Y. Wang, RSC Adv. 2014, 4, 6493664946; c) Ziarani, G. M.; Hasankiadeh, F. S.; Mohajer, F. ChemistrySelect 2020, 5, 14349-14379; d) Chen, M.-E.; Chen, X.-W.; Hu, Y.-H.; Ye, R.; Lv, J.-W.; Li, B.; Zhang, F.-M. Org. Chem. Front. 2021, DOI: 10.1039/D1QO00496D.

[14] C. Liu, F. Yang, Eur. J. Org. Chem. 2019, 6867-6870. 
[15] V. V. Zhdankin, C. J. Kuehl, A. P. Krasutsky, J. T. Bolz, A. J. Simonsen, J. Org. Chem. 1996, 61, 6547-6551.

[16] a) W. Ding, J. Chai, C. Wang, J. Wu, N. Yoshikai, J. Am. Chem. Soc. 2020, 142, 8619-8624; b) J. Chai, W. Ding, J. Wu, N. Yoshikai, Chem. Asian J. 2020, 15, 2166-2169.

[17] A. Saito, A. Taniguchi, Y. Kambara, Y. Hanzawa, Org. Lett. 2013, 15, 2672-2675.

[18] a) Y. Li, D. P. Hari, M. V. Vita, J. Waser, Angew. Chem. Int. Ed. 2016 55, 4436-4454; b) J. Waser, Synlett 2016, 27, 2761-2773; c) D. P. Hari, P. Caramenti, J. Waser, Acc. Chem. Res. 2018, 51, 3212-3225; d) N Declas, G. Pisella, J. Waser, Helv. Chim. Acta 2020, 103, e2000191.

[19] Pozhydaiev, V.; Power, M.; Gandon, V.; Moran, J.; Lebœuf, D. Chem. Commun. 2020, 56, 11548-11564.

[20] a) J. Wu, X. Deng, H. Hirao, N. Yoshikai, J. Am. Chem. Soc. 2016, 138, 9105-9108; b) B. Wu, J. Wu, N. Yoshikai, Chem. Asian J. 2017, 12, 31233127; c) J. Wu, K. Xu, H. Hirao, N. Yoshikai, Chem. Eur. J. 2017, 23 1521-1525; d) W. Ding, C. Wang, J. R. Tan, C. C. Ho, F. Leon, F. Garcia, N. Yoshikai, Chem. Sci. 2020, 11, 7356-7361.

[21] a) E. Stridfeldt, A. Seemann, M. J. Bouma, C. Dey, A. Ertan, B. Olofsson Chem. Eur. J. 2016, 22, 16066-16070; b) P. Caramenti, N. Declas, R. Tessier, M. D. Wodrich, J. Waser, Chem. Sci. 2019, 10, 3223-3230; c) L. Castoldi, E. M. Di Tommaso, M. Reitti, B. Grafen, B. Olofsson, Angew. Chem. Int. Ed. 2020, 59, 15512-15516; d) N. Declas, J. Waser, Angew. Chem. Int. Ed. 2020, 59, 18256-18260.

[22] a) M. Movassaghi, M. D. Hill, O. K. Ahmad, J. Am. Chem. Soc. 2007, 129 10096-10097; b) M. Movassaghi, M. D. Hill, J. Am. Chem. Soc. 2006 $128,14254-14255$ 


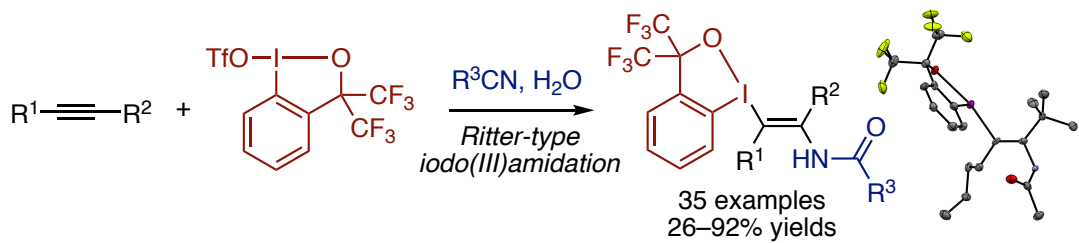

A Ritter-type trans-difunctionalization of alkynes is promoted by benziodoxole triflate, a trivalent iodine electrophile, thus affording tetrasubstituted, $\beta$-iodanyl enamides in moderate to good yields. The reaction is applicable to various internal alkynes and nitriles, and transformation of the iodanyl group allows for the preparation of tri- and tetrasubstituted enamides that are difficult to synthesize by other existing methods. 